\title{
Obtaining Pulsatile Flow Rate Using a 360-Degree Bend
}

\author{
Masaru Sumida \\ Faculty of Engineering, Kinki University, Japan
}

Copyright (C) 2015 Horizon Research Publishing All rights reserved.

\begin{abstract}
360-degree bend flowmeters were applied to determine pulsatile flow rates in water. Experiments were performed using bend flowmeters with curvature radius ratios of 7, 10 and 30 under the conditions of Womersley numbers of $\alpha=2.1-18$, mean Reynolds numbers of $R e_{t a}=800-5 \times 10^{4}$ and oscillatory Reynolds numbers of $R e_{o s}=600-1.6 \times 10^{4}$. The pressure difference across the bend was found to be independent of the pulsation frequency and to conveniently vary with almost the same phase as the instantaneous flow rate. A method for measuring the instantaneous flow rate is proposed as well as a method for estimating the time-mean and amplitude values of the pulsatile flow rate. It is concluded that 360-degree bend flowmeters can be used to measure the pulsatile flow rate.
\end{abstract}

Keywords Flow Measurement, Unsteady Flow, Bend Flowmeter, Pulsatile Flow, Discharge Coefficient, Curved Pipe

\section{Introduction}

In various plants, piping systems and fluid machines and devices, it is essential to know the flow rate and the state of flow at any time. Therefore, many methods of performing fluid measurements have been rapidly developed and modified. However, as seen from restriction flowmeters, long-term efforts to develop methods of performing flow measurements have focused on steady pipe flows. On the other hand, the flows in pipes are frequently unsteady, such as the flows discharged from a rotary and a bellows pump or emitted from a reciprocating engine. Moreover, the gas in an oxygenator and the blood in arteries are also unsteady and time-dependent periodic flows. Therefore, several studies have been carried out to obtain unsteady flow rates using conventional equipment [1-3]. Nevertheless, a number of problems remain to be solved [4].

It should be noted that bend and elbow flowmeters can be used to measure the flow rate by installing them in pipelines. The bend flowmeter is a convenient and economical device for flow measurements [5] and its various uses have been investigated over many years [6-8]. Studies have been carried out on bend flowmeters with several turn angles, in which the effects of the radius ratio and Reynolds numbers on the discharge coefficients have been investigated intensively. The state of research, including that on the flow conditions, was described in detail by Ito and Miyakawa [9]. Moreover, the effect of the forming accuracy of the bend on the discharge coefficients has been examined [10, 11]. In addition, slurries and other problem fluids have also been taken up as a topic of research $[12,13]$.

However, as mentioned above, steady flows were chosen as the subject of these studies. Therefore, to the author's knowledge, unsteady flows have not yet been studied in depth, even though the necessity of their research was first pointed out over 50 years ago [14, 15]. Moreover, investigations concerned with characteristics of unsteady flows of the curved pipes relevant to the shape of bend flowmeters are limited to laminar flows with relatively low Reynolds numbers [16, 17]. Research on practicable turbulent flows in industry, on the other hand, is extremely few $[18,19]$. In these studies [18, 19], velocity distributions in pulsating turbulent flows were measured by laser Doppler velocimetry for a $180^{\circ}$ curved duct with $R_{c}=5$ [18] and a $90^{\circ}$ bend with $R_{c}=4$ [19]. In unsteady flows, such as time-dependent periodic flows, it is necessary to accurately determine not only the time mean but also the amplitude of the varying component of the flow rate [4].

With regard to obtaining the instantaneous flow rate of an unsteady pipe flow with pulsation, the following two methods are well known. One is the direct use of a flowmeter, such as an electromagnetic flowmeter or an ultrasonic velocity profiler, with high responsibilities. The other is a method of obtaining the time-dependent flow rate by correcting a read value using the dynamic characteristics of a flowmeter [20]. The former method has the disadvantage that it is expensive to install a flowmeter at several locations in pipelines. Hence, except for special cases, the latter method has generally been adopted in industry. However, when restriction and area flowmeters are to be installed in pipelines, the mounting position is sometimes restricted by the length of the straight inflow pipe and by the direction.

In this paper, we describe the pressure characteristics of a pulsatile flow in 360-degree (i.e., full-circle pipe) bend flowmeters [9, 14, 21, 22] and propose a method of measuring the pulsatile flow rate using such flowmeters. In $360^{\circ}$ bend flowmeters, a fully developed curved-pipe flow 
can be effectuated, regardless of the velocity distribution at the entrance and the turn angle in the measuring section. In addition, $360^{\circ}$ bend flowmeters can be installed in part of a straight pipeline. According to previous studies [14, 22] on steady flows, the $360^{\circ}$ bend flowmeter enables more stable measurements than $90^{\circ}$ and $180^{\circ}$ bend flowmeters. Thus, first of all, it is very important to examine the characteristics of a pulsatile flow in a $360^{\circ}$ bend flowmeter in consideration of its applicability to measurements of the unsteady flow rate.

\section{Nomenclature}

Please also refer to Fig. 4, as hereinafter described, which shows the coordinate system and the dimensions of the bend in this study.

$a$ : pipe radius, $=d / 2$

$C_{d}$ : discharge coefficient, equation (2)

$d$ : pipe diameter

$E$ : correction factor, equation (17)

$h$ : differential pressure head across pipe cross section, $=\left(p_{o}-p_{i}\right) /(\rho g)$

$p$ : pressure

$p_{o}, p_{i}:$ pressures on outside and inside walls of pipe

$q$ : flow rate

$R$ : curvature radius at centerline of pipe

$R_{c}$ : curvature radius ratio, $=R / a$

$R e$ : Reynolds number, $=w_{m} d / v$

$R e_{o s}:$ oscillatory Reynolds number, $=w_{m, o s} d / v$

$R e_{t a}:$ mean Reynolds number, $=w_{m, t a} d / v$

$R_{h}$ : Reynolds number with differential pressure head $h$, $=(2 g h)^{1 / 2} d / v$

$R_{h, t a}$ : Reynolds number with time-mean differential pressure head $h_{t a},=\left(2 g h_{t a}\right)^{1 / 2} d / v$

$u, v, w$ : velocities in $x, y$ and $z$ directions

$x, y, z$ : coordinate system

$\alpha$ : Womersley number (nondimensional frequency), $=a(\omega / v)^{1 / 2}$

$\beta$ : ratio of pressure-head difference, $h_{t a} / h_{s}$ $\eta$ : flow rate ratio, $=w_{m, o s} / w_{m, t a}$

$\eta_{h}$ : amplitude ratio of differential pressure head, $=h_{o s} / h_{t a}$ $\Theta$. phase angle, $=\omega t$ ( $t$ : time, $\omega$ : angular frequency)

$v, \rho$ : kinematic viscosity and density of fluid

$\Phi$ : phase difference

Subscripts

$m$ : cross-sectional averaged value

$s$ : steady flow

ta, os: time-mean and amplitude values

\section{Experimental Apparatus and Procedure}

A schematic diagram of the experimental apparatus is shown in Fig. 1. The system consists of a pulsatile flow generator, a test $360^{\circ}$ bend flowmeter, a backpressure control tank and devices for measuring the differential pressure. The working fluid was water. The pulsatile flow generator was composed of a steady flow and an oscillatory flow generator. The steady flow was supplied from a head tank or by a pump. The volume-cycled oscillatory flow was generated by a reciprocating piston pump installed in a scotch yoke mechanism, which was driven by a variablespeed motor. The piston pump can be chosen from those with diameters of 0.6 to $4 \mathrm{~cm}$ and its stroke was adjustable in the range of 0 to $10 \mathrm{~cm}$. Thus, a pulsatile flow having a sinusoidal waveform with a prescribed amplitude and frequency can be effectuated in the test bend [23], 1984). That is, the pulsating flow rate $q$ in the test bend flowmeter can be expressed in the form

$$
q=q_{t a}+q_{o s} \sin \Theta=q_{t a}(1+\eta \sin \Theta) .
$$

Here, $\Theta(=\omega t)$ is the phase angle, $\omega$ is the angular frequency and $t$ is the time. Subscripts $t a$ and $o s$ indicate the time mean and amplitude, respectively.

Three bends, with dimensions shown in Tab. 1, were employed as the test flowmeters in the present study. They were made of accurately machined transparent acrylic plates. The dimensions were chosen with reference to $360^{\circ}$ bend flowmeters examined in previous works [9, 14, 21, 22] on steady flows; the dimensions tested are shown in Tab. 2. The relative roughness of the inside of the bend, which was milled by an NC cutter, was $10^{-4}$ and the skewness of the inside diameter out of roundness was $0.2 \%$ or less. Hence, the test bend flowmeters were finished to a very hydraulically smooth surface and were also geometrically accurate. The position of the pressure measurement was set at the turn angle of $180^{\circ}$ measured from the bend entrance with reference to the results of previous studies [9, 14, 21, 22] on steady flows. Furthermore, the diameter of the pressure holes was $0.4 \mathrm{~mm}$ on the basis of the results of studies on steady flows $[9,24]$ and on pulsatile flows in straight pipes [23]. The pressure difference between the outside and inside of the bend was detected by a diffusive-type semiconductor pressure transducer (Toyoda MFG, DD102A-0.1F and 0.3F), which has a frequency characteristic of about $600 \mathrm{~Hz}$ in air. The pressure transducer was connected almost symmetrically to the measuring taps with the stainless pipes as shown in Fig. 2. The outputs from the transducer were amplified by the DC amplifier and were recorded through a low-pass filter for about ten pulsation cycles. Simultaneously, a time-marker signal that was generated by an electro-optical device was also recorded to determine the pulsation frequency. Then the recorded data were processed with a computer. 


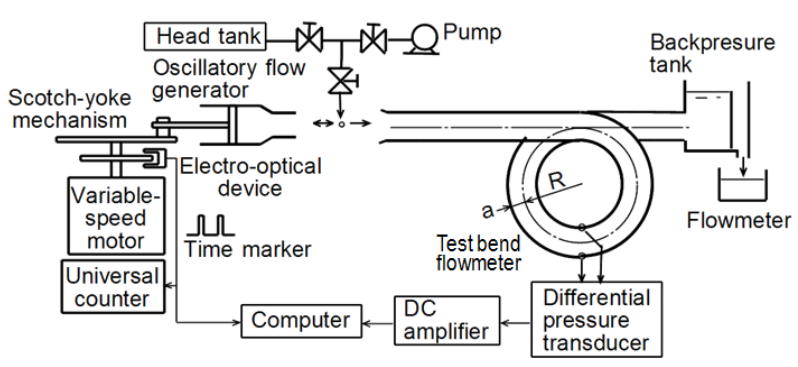

Figure 1. Schematic diagram of apparatus for bend flowmeter tests

Table 1. Dimensions of test bend flowmeters

\begin{tabular}{|c|c|c|c|}
\hline No & $\begin{array}{c}\text { Diameter } \\
d \mathrm{~mm}\end{array}$ & $\begin{array}{c}\text { Curvature radius } \\
R \mathrm{~mm}\end{array}$ & $\begin{array}{c}\text { Curvature radius } \\
\text { ratio } R c\end{array}$ \\
\hline 1 & 8.0 & 28.0 & 7.0 \\
\hline 2 & 8.0 & 40.0 & 10.0 \\
\hline 3 & 8.0 & 120.0 & 30.0 \\
\hline
\end{tabular}

Table 2. Test bend flowmeters employed in previous works on steady flows

\begin{tabular}{|c|c|c|}
\hline Author & Diameter $d \mathrm{~mm}$ & $\begin{array}{c}\text { Curvature radius } \\
\text { ratio } R c\end{array}$ \\
\hline Cortelyou [14 ] & $19.05,25.4$ & $6,9.33$ \\
\hline Tsuji \& Kawashima [21] & $7.4 \sim 17.0$ & $6.78 \sim 10.0$ \\
\hline Ito \& Miyakawa [9] & $16.09 \sim 35.07$ & $7.02 \sim 27.9$ \\
\hline Ito et al. [22] & $35.05 \sim 88.94$ & $10.01 \sim 27.97$ \\
\hline
\end{tabular}

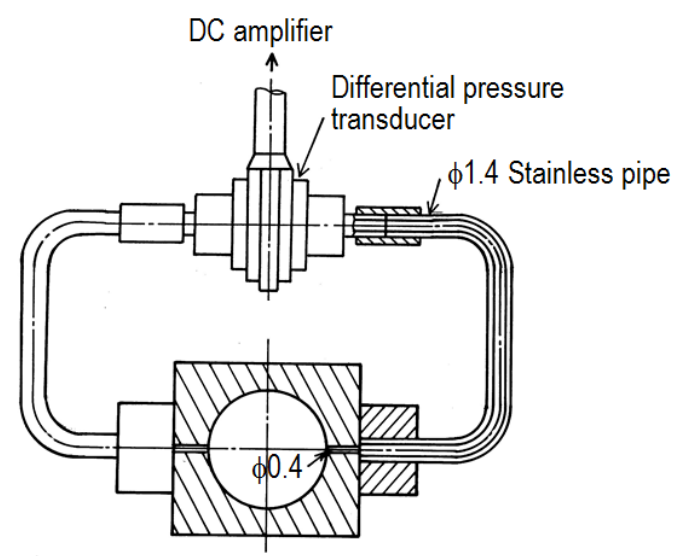

Figure 2. Arrangement of pressure transducer and test bend flowmeter.

The experiments were performed systematically under the conditions of Womersley numbers $\alpha$ of 2.1 to 18 (frequencies of $0.05-3.6 \mathrm{~Hz}$ ), mean Reynolds numbers $R e_{t a}$ of 800 to $5 \times 10^{4}$ and oscillatory Reynolds numbers $R e_{o s}$ of 600 to 16000 (flow rate ratios $\eta=0.03-3.5$ ). Here, the Womersley number is defined as $\alpha=a(\omega / v)^{1 / 2}$ with $a$ being the pipe radius and $v$ being the kinematic viscosity of the fluid. The mean and oscillatory Reynolds numbers are expressed as $R e_{t a}=w_{m, t a} d / v$ and $R e_{o s}=w_{m, o s} d / v$, respectively, where $w$ is the axial velocity and the subscript $m$ indicates the cross-sectional average value. Moreover, the flow rate ratio $\eta$ is given by $\eta=w_{m, o s} / w_{m, t a}$. These conditions were chosen in consideration of industrial use and flows in bioengineering. The measured pressure difference was decomposed into time-mean and amplitude values and the phase difference between the variations of the pressure difference and the flow rate was obtained. Thus, the response characteristic of the $360^{\circ}$ bend flowmeters to the varying pressure difference was examined.

\section{Results and Discussion}

The relations between the discharge coefficient $C_{d}$ and Reynolds number $R e$ in the steady flow are shown in Fig. 3. $C_{d}$ is defined by the following form [24] in terms of the differential pressure head $h$ :

$$
q=C_{d} A(2 g h)^{1 / 2} .
$$

Here, $h=\left(p_{0}-p_{i}\right) /(\rho g)$, where $p_{0}$ and $p_{i}$ are the pressures at the outside and inside walls of the cross section, respectively, $\rho$ is the density of the fluid and $g$ is gravitational acceleration. Moreover, $A$ is the cross-sectional area of the bend. The experimental results agree well with the empirical formulas of Ito and Miyakawa [9], which are indicated by broken and solid lines:

$$
\begin{gathered}
C_{d}=f_{1}-f_{2} R_{h}^{-1 / 2} \quad \text { (laminar flow), } \\
C_{d}=g_{1}-g_{2} R_{h}^{-1 / 5} \quad \text { (turbulent flow), }
\end{gathered}
$$

where $R_{h}$ is the Reynolds number defined by

$$
R_{h}=(2 g h)^{1 / 2} d / v\left(=R e / C_{d}\right)
$$

and

$$
\left.\begin{array}{l}
f_{1}=0.453 R_{c}^{1 / 2}+0.16 R_{c}^{-1 / 2}, \\
f_{2}=0.51 R_{c}^{1 / 2}, \\
g_{1}=0.512 R_{c}^{1 / 2}+0.78 R_{c}^{-2}, \\
g_{2}=0.070 R_{c}^{4 / 5} .
\end{array}\right\}
$$

In the above expressions, $R_{c}$ is the curvature radius ratio $R / a$.

$C_{d}$ generally increases with increasing Reynolds number. When the Reynolds number becomes large, the discharge coefficient can be approximated by the following expression, as indicated by the dash-dotted lines in Fig. 3,

$$
C_{d}=R_{c}^{1 / 2} / 2 .
$$

This formula was derived by assuming the fluid motion to be a forced vortex flow [7].

In the following, first assuming the velocity distribution of the forced vortex flow, we estimate the changes in the differential pressure of the pulsatile flow and clarify their characteristics. Subsequently, we describe the method of obtaining the pulsatile flow rate from the measured differential pressure using the characteristics. 


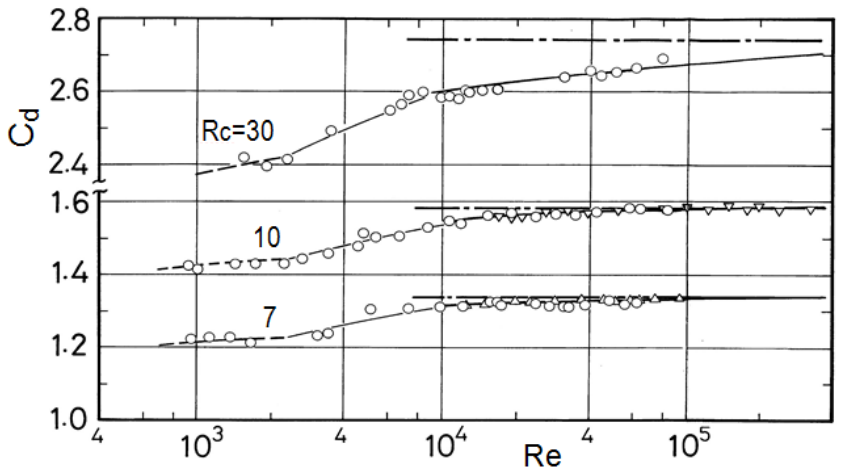

Figure 3. Discharge coefficients for steady flow. $\circ$, present results; $\Delta$ and $\nabla$, previous data for $R c=7.02$ and 9.97 , respectively [9, 22]. Broken and solid lines: empirical formulas of Ito and Miyakawa [9], equations (3) and (4), respectively. Dash-dotted lines: equation (8).

\subsection{Changes in Differential Pressure and their Characteristics}

We consider the flows at the central part of the cross section except for the region near the pipe wall where the effects of the viscosity of the fluid are large. In this study, the orthogonal curvilinear coordinates $(x, y, z)$ are adopted as shown in Fig. 4. The equation of motion of an inviscid and incompressible fluid in the $x$-direction can be written for a fully developed flow as

$$
\frac{1}{\rho} \frac{\partial p}{\partial x}=\frac{w^{2}}{R+x} .
$$

In this equation, the secondary flow velocities $u$ and $v$ are neglected because they are very small compared with the axial velocity $w$. When referring to the measurement results [25] shown in Fig. 5, we introduce the axial velocity $w$ in the forced vortex distribution as

$$
w=w_{m}[1+f(\Theta) \cdot x / R] .
$$

Here, $w_{m}$ is the cross-sectional average velocity, $w_{m}=w_{m, t a}$ $(1+\eta \sin \Theta)$ and $f(\Theta)$ is a function of the phase angle $\Theta$. Substituting (10) into (9) and integrating it, we obtain the pressure difference between the outside $\left(\theta=90^{\circ}\right)$ and inside walls $\left(\theta=-90^{\circ}\right)$ as

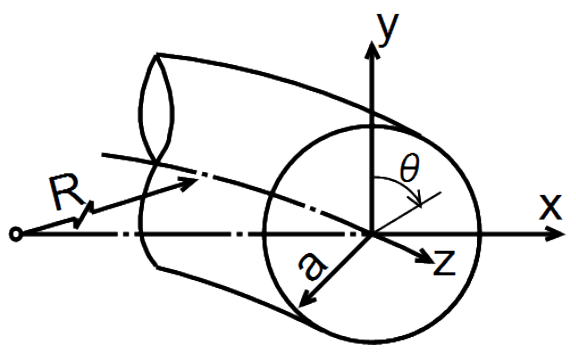

Figure 4. Coordinate system

$$
\begin{aligned}
& \frac{1}{\rho}\left(p_{o}-p_{i}\right)=\int_{-a}^{a} \frac{w^{2}}{R+x} d x \\
&= w_{m}^{2}\left[(1-f)^{2} \log \left(\frac{1+\varepsilon}{1-\varepsilon}\right)+2 f(2-f) \varepsilon\right] \\
& \varepsilon\left(=1 / R_{c}\right)<1
\end{aligned}
$$

Expanding the logarithmic function as a power series, the above equation is rewritten as

$$
\begin{aligned}
\frac{1}{\rho}\left(p_{0}-p_{i}\right)=w_{m}^{2} & {\left[2 \frac{a}{R}+O\left(\varepsilon^{3}\right)\right] } \\
=\left[\left(1+\frac{\eta^{2}}{2}\right) w_{m, t a}^{2}\right. & \left.+2 \eta w_{m+a}^{2} \sin \Theta+\frac{\eta^{2}}{2} w_{m \neq a}^{2} \sin \left(2 \Theta-\frac{\pi}{2}\right)\right] \\
\times & {\left[2 \frac{a}{R}+O\left(\varepsilon^{3}\right)\right] . }
\end{aligned}
$$

In the following, we consider the time-mean and amplitude values of the pressure difference and its phase difference from the flow rate. Then, we investigate their characteristics by comparing the experimental results with the approximate results obtained using (12).

First, we examine the time-mean pressure difference, $\left(p_{o}-p_{i}\right)_{t a}=\rho g h_{t a}$, in comparison with $\left(p_{o}-p_{i}\right)_{s}=\rho g h_{s}$ in a steady flow with the same flow rate as the time-mean flow rate in the pulsatile flow. The ratio of the two pressure differences is denoted by $\beta$, i.e.,

$$
\beta=\left(p_{o}-p_{i}\right)_{t a} /\left(p_{o}-p_{i}\right)_{\mathrm{s}}=h_{t a} / h_{s} .
$$

Then, substituting (12) into (13) and disregarding the terms of $\varepsilon^{3}$ or more, as a first approximation, we obtain the ratio $\beta$ as

$$
\beta=1+\eta^{2} / 2
$$

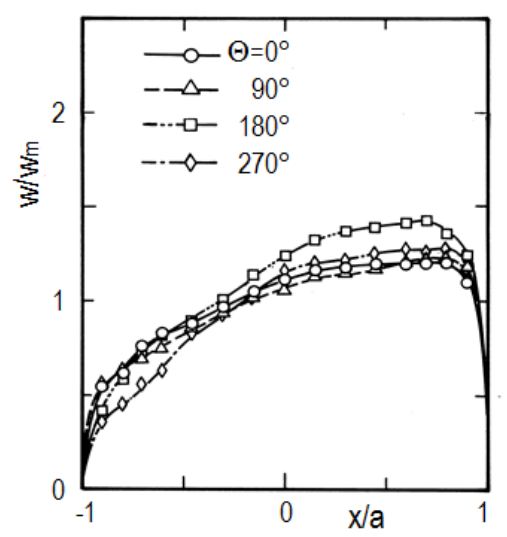

Figure 5. Example of axial velocity distribution measured by LDV [25]. $\left(\alpha=18.7, \operatorname{Re}_{t a}=34200, \operatorname{Re}_{o s}=16700, R_{c}=11.2\right)$ 

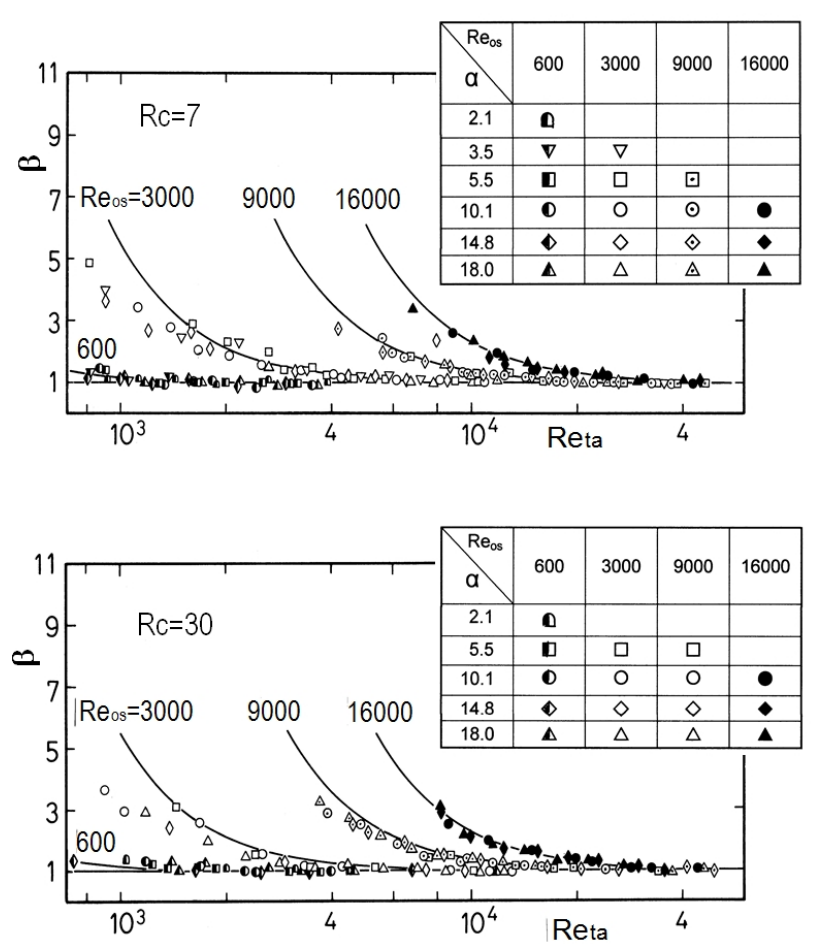

Figure 6. Time mean of differential pressure head. Relationship between $\beta$ and $R_{t a}$. Solid lines: equation (14).

Consequently, the ratio $\beta$ can be expressed in terms of $\eta$, which is one of the governing parameters of the pulsatile flow in curved pipes [23]. Figure 6 shows the experimental results for $\beta$ with various $\alpha$ at a constant $R e_{o s}$ for bends with $R_{c}=7$ and 30 , where the solid lines denote (14). The time-mean pressure difference is almost independent of $\alpha$. Although it is larger than that in the steady flow for $R e_{t a}$ below a certain number, $\beta$ approaches one with increasing $R e_{t a}$. The agreement with the approximate (14) is good in the flows with $R e_{t a} \geq \operatorname{Re}_{o s}(1 \geq \eta)$. However, when $\eta$ becomes large for a low $R e_{t a}$, the experimental results are smaller than those estimated from (14).

Figure 7 shows waveforms of the instantaneous differential pressure head $h$ for an $R e_{o s}$ of about 3000. In Fig. 7 , the data are plotted using the method of moving averages with five values. Moreover, the solid lines indicate the first fundamental component obtained from the Fourier series, while the broken lines show the experimental results $h_{s}$ for the steady flow with the same Reynolds number $R e_{t a}$. The variation of the pressure difference increases in proportion to the product of $w_{m, t a}$ and $w_{m, o s}$ as indicated by (12). That is, the variation of $h$ rapidly increases as the time-mean flow rate increases, even if the amplitude of the flow rate is fixed. Additionally, the differential pressure head $h$ can be expressed approximately, except for a certain part of the period for large $\eta$, by the first fundamental component in the Fourier expansion.

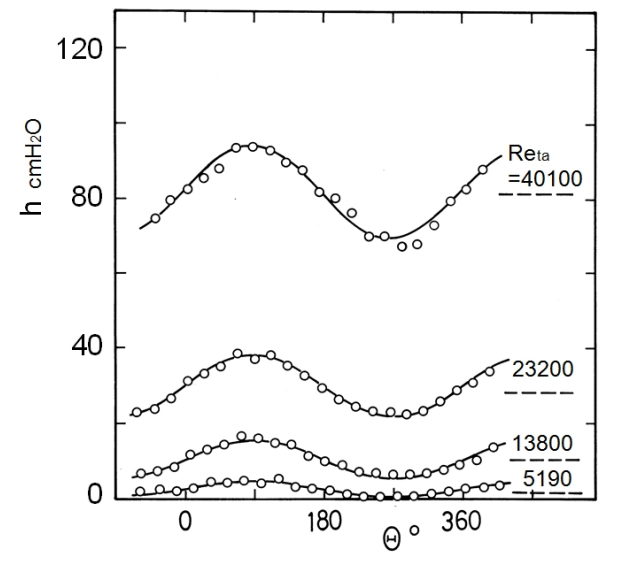

Figure 7. Effect of $R e_{t a}$ on changes in differential pressure head $h$ with time. $\alpha=14.8 \pm 0.1, \operatorname{Re}_{o s}=(3.0 \pm 0.15) \times 10^{3}, R_{c}=7$. Solid lines: fundamental component of Fourier expansion. Broken lines: steady flow.

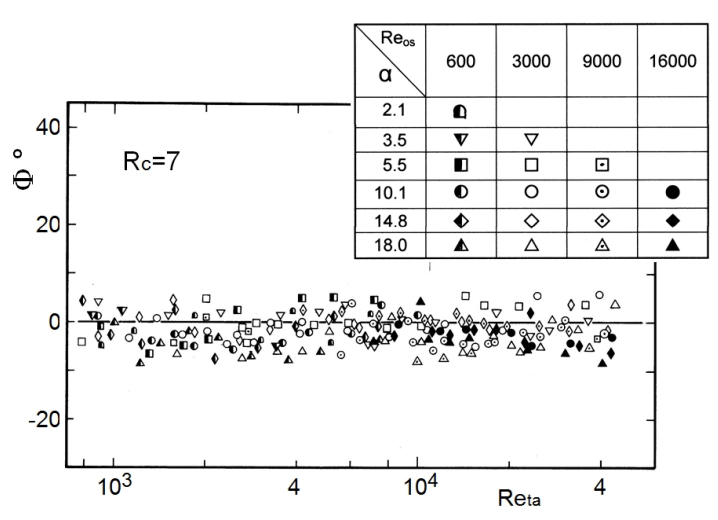

Figure 8. Phase difference $\Phi$ of differential pressure head $h$ from flow rate.

Thus, we examine the first fundamental component of the waveform of the pressure difference. Examples of the phase difference $\Phi$ from the flow rate are shown in Fig. 8. The experimental results show a phase difference of approximately zero regardless of the values of $R_{c}, R e_{t a}$ and $\eta$, although they vary within $\pm 6^{\circ}$. Consequently, it can be considered that the pressure difference changes almost in phase with the flow rate.

From the above observations, the magnitude of the pressure-difference variation can be expressed using the results of the approximate analysis, assuming the velocity distribution of a forced vortex flow for flows with $R e_{t a}>R e_{o s}$ $(1 \geq \eta)$. Furthermore, it is practically acceptable to consider that the pressure difference changes almost in phase with the flow rate. In the following paragraphs, we will describe how to actually obtain the pulsatile flow rate on the basis of the results in this section. 


\subsection{Procedure for Obtaining Pulsatile Flow Rate by Measuring Instantaneous Pressure Difference}

Using the characteristic that there is little phase difference between $h$ and $q$, we apply (2) to obtain an instantaneous flow rate using the bend flowmeter. Figure 9 shows a comparison of the instantaneous flow rates obtained from (2) with those provided by (1). In Fig. 9(a), the plots indicate the former flow rates obtained using the discharge coefficient from (8), while the solid line displays the latter flow rates. The measured pressure difference is shown in Fig. 9(b), where the solid line indicates the fundamental component of the Fourier expansion. The measured flow rate is in good agreement with that generated by the pulsatile flow generator for the period when the flow rate is comparatively large, i.e., $q \geq 50 \mathrm{~cm}^{3} / \mathrm{s}$ $(R e \geq 8000)$. Similar results were obtained for flows with other values of $\alpha$ and $\eta$. Therefore, when $R e \geq 8000$, the instantaneous flow rate can be measured with reasonably good accuracy using the present bend flowmeter by applying $C_{d}$ obtained from (8).

On the other hand, for periodically unsteady flows, there are some cases where it is sufficient to know the time mean $q_{t a}$ and amplitude $q_{o s}$ of the flow rate. In these cases, we use the characteristic that the waveform of the pressure difference can be satisfactorily expressed by the fundamental component of the Fourier expansion. That is, we write the measured differential pressure head $h$ as

$$
h \approx h_{t a}+h_{o s} \sin \Theta=h_{t a}\left(1+\eta_{h} \sin \Theta\right) .
$$

Here, we describe a method to easily obtain the values of $q_{t a}$ and $q_{o s}$ of the flow rate $q$ on the basis of the relationship given by (2) as shown in Fig. 9. We obtain the time-averaged flow rate by integrating $q$ over a period as

$$
q_{t a}=\frac{1}{2 . \pi} \int_{0}^{2 \pi} C_{d} A \sqrt{2 g h} d \Theta .
$$

(b)

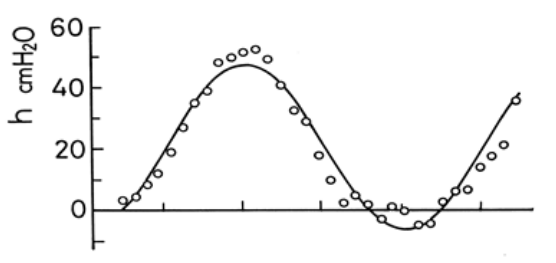

(a)

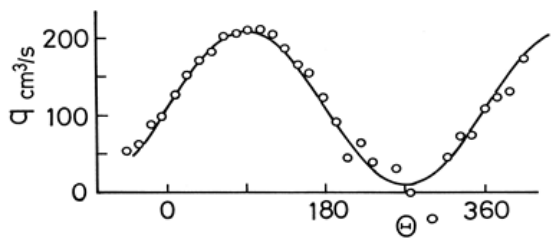

Figure 9. Example of measured instantaneous flow-rate. $\alpha=17.9$, $R e_{t a}=17400, \eta=0.90$ for $R_{c}=7$. Figure (a) shows the flow rate obtained from the measured pressure head $h$ in fig. (b). Solid lines in figs. (a) and (b) indicate equation (1) and fundamental component of Fourier expansion, respectively.

Substituting (15) into the above equation, we obtain

$$
q_{t a}=E C_{d} A \sqrt{2 g h_{t a}} .
$$

Here, $E$ is the correction factor when the average flow rate is calculated from the measured $h_{t a}$. Using $C_{d}$ from (8), it becomes

$$
E(=k) \approx 1-\frac{1}{16} \eta_{h}{ }^{2}-\frac{15}{1024} \eta_{h}{ }^{4}
$$

(forced vortex flow).

In the same manner, adopting the empirical formulas (3) and (4) [9] for $C_{d}$, we derive the correction factors

$$
\begin{gathered}
E \approx k\left(1-\frac{k_{1}}{k} \frac{f_{2}}{f_{1}} R_{h, t a}^{-1 / 2}\right) /\left(1-\frac{f_{2}}{f_{1}} R_{h, t a}^{-1 / 2}\right) \\
\text { (laminar flow), (19) } \\
E \approx k\left(1-\frac{k_{2}}{k} \frac{g_{2}}{g_{1}} R_{h, t a}^{-1 / 5}\right) /\left(1-\frac{g_{2}}{g_{1}} R_{h, t a}^{-1 / 5}\right) \\
\text { (turbulent flow), }
\end{gathered}
$$

where

$$
\left.\begin{array}{l}
k_{1} \approx 1-\frac{3}{64} \eta_{h}^{2}-\frac{231}{16384} \eta_{h}^{4} \\
k_{2} \approx 1-\frac{3}{50} \eta_{h}^{2}-\frac{39}{2500} \eta_{h}^{4}
\end{array}\right\}
$$

In (19) and (20), $R_{h, t a}$ is the Reynolds number with a time-mean differential pressure head $h_{t a}$ and is defined as $\left(2 g h_{t a}\right)^{1 / 2} d / v$. Figure 10 shows the relationship between the correction factor $E$ and the amplitude ratio $\eta_{h}$ of the differential pressure waveform. The factors obtained from (19) and (20) agree with the value given by (18) within $0.1 \%$. Consequently, it is reasonable to practically apply (18), analytically derived for a forced vortex flow, to the correction factor.

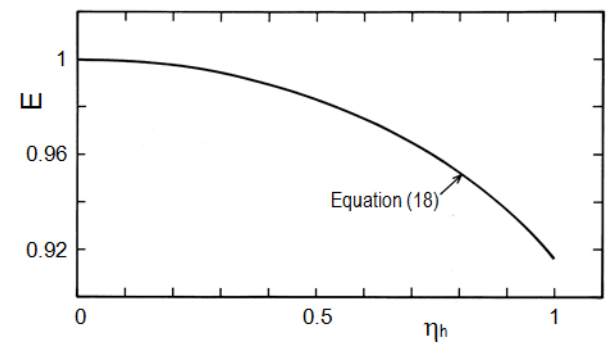

Figure 10. Relationship between correction factor $E$ and amplitude ratio $\eta_{h}$ of differential pressure head.

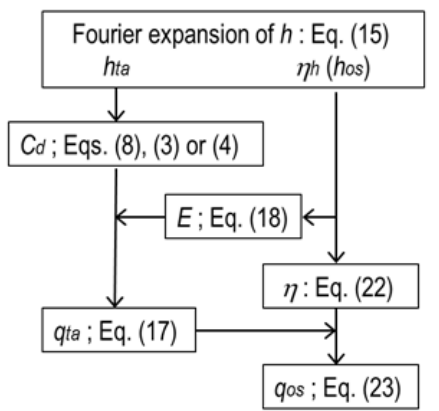

Figure 11. Procedure for simplified method of measuring $q_{t a}$ and $q_{o s}$. 


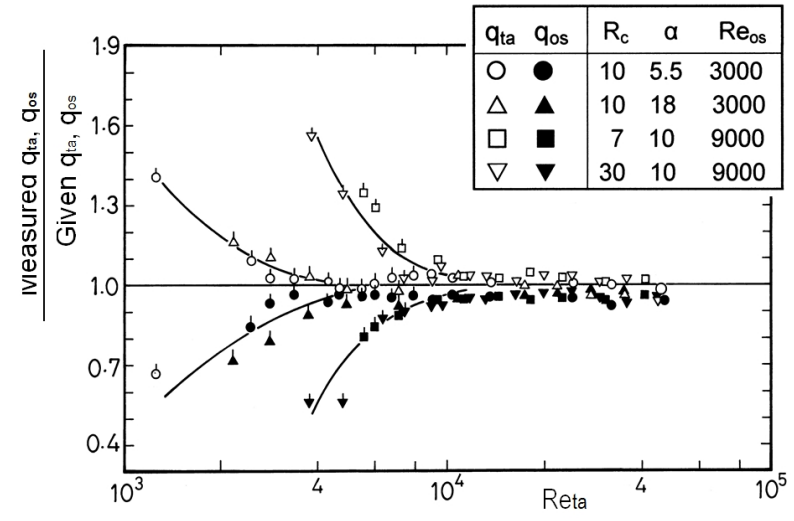

Figure 12. Comparison of measurement values obtained by simplified method and prescribed values of $q_{t a}$ and $q_{o s}$. Marked symbols denote flow rates obtained by using equation (3) as $C_{d}$.

Note that using (12), the flow rate ratio $\eta$ can be rewritten in terms of the amplitude ratio $\eta_{h}$ of the differential pressure waveform as follows:

$$
\eta\left(=q_{o s} / q_{t a}\right)=\left\{2-\left(4-2 \eta_{h}^{2}\right)^{1 / 2}\right\} / \eta_{h} .
$$

Accordingly, the oscillatory component $q_{o s}$ of the flow rate is obtained as

$$
q_{o s}=\eta q_{t a} .
$$

The above-mentioned procedure is summarized in Fig. 11. Figure 12 shows the ratio of the flow rates, $q_{t a}$ and $q_{o s}$, obtained from the $h$ waveform by applying this procedure to the prescribed flow rates given by (1). Here, with regard to $C_{d}$, Equation (8) is used for flows with $R e_{t a} \geq 8000$, and the empirical formula (3) [9] is used for flows with $R e_{t a} \leq 8000$. The measured flow rate approaches that of the flow driven by the flow generator as the mean Reynolds number increases. For flows with $R e_{t a} \geq 1.5 R e_{o s}$, i.e., $\eta_{h} \leq 1.09$ ( $\eta$ $\leq 0.67)$, the measured values of $q_{t a}$ and $q_{o s}$ agree with those for the flow driven by the flow generator within 4 and $6 \%$, respectively. Therefore, we can conclude that the present method is convenient for determining the pulsatile flow rate. However, when the flow rate ratio $\eta$ exceeds one, the accuracy of the calculation of $E$ and $\eta$, and consequently that of the measured flow rate, decreases. This is because $\eta_{h}$ decreases as the period during which the fluid flows in the opposite direction increases.

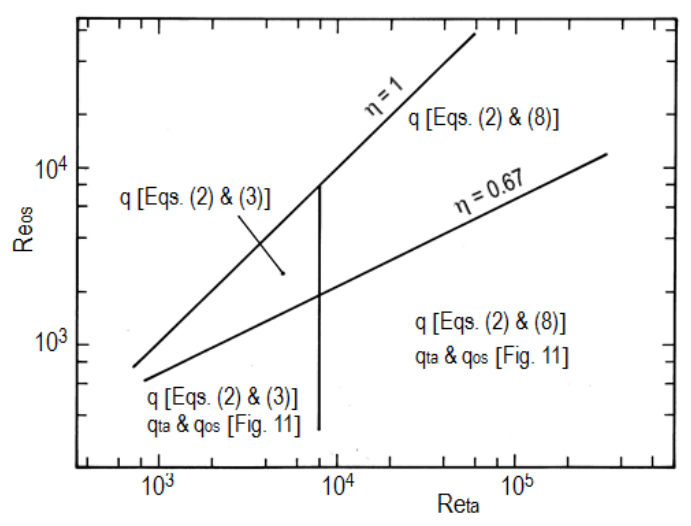

Figure 13. Measurable quantities using the proposed method for the bend flowmeter.
Figure 13 demonstrates the quantities, i.e., $q, q_{t a}$ and $q_{o s}$, that can be measured using the proposed method for the bend flowmeter, and the ranges of the Reynolds numbers in which they can be measured. For this type of flowmeter, flows with Reynolds numbers of $10^{4}$ to $10^{5}$ are important in industry. Furthermore, the instantaneous flow rate for the period when $R e \leq 8000$ can be accurately obtained by (2), in which (3) for the laminar flow is adopted as the discharge coefficient.

\section{Conclusions}

The characteristics of the differential pressure across a bend were investigated experimentally for pulsatile flows under the conditions of $\alpha=2.1-18, R e_{t a}=800-5 \times 10^{4}$ and $R e_{o s}=600-1.6 \times 10^{4}(\eta \leq 3.5)$, and the applicability of a bend flowmeter to pulsatile flow rate measurements was examined. The principal achievements and results of this study are summarized as follows.

(1) The effect of the pulsation frequency $(0.05-3.6 \mathrm{~Hz})$ on the detected differential pressure of a $360^{\circ}$ bend flowmeter did not appear within the ranges of $7 \leq R_{c}$ $\leq 30$ and $800<R e_{t a}<5 \times 10^{4}$. It was found that the differential pressure changes almost in phase with the variation in the flow rate and is suitable for measuring the pulsatile flow rate. Moreover, the differential pressure can be expressed using the results of an approximate analysis in which the velocity distribution of a flow with $\eta_{h}<1$ is assumed to be that of a forced-vortex flow.

(2) Currently available bend flowmeters can be used to measure the instantaneous flow rate, which is obtained from (2) and (8) with the instantaneous differential pressure head when $R e \geq 8000$ or $q \geq 50$ $\mathrm{cm}^{3} / \mathrm{s}$.

(3) For flows with $\eta \leq 0.67\left(\eta_{h} \leq 1.09\right)$, the time mean and amplitude of the pulsatile flow rate, $q_{t a}$ and $q_{o s}$, respectively, can be obtained easily from the amplitude ratio $\eta_{h}$ of the differential pressure head.

\section{REFERENCES}

[1] Durst, F., Heim, U., Unsal, B. and Kullik, G., Mass flow rate control system for time-dependent laminar and turbulent flow investigations, Measurement Science and Technology, Vol. 14, No. 7 (2003), pp. 893-902.

[2] Brereton, G. J., Schock, H. J. and Bedford, J. C., An indirect technique for determining instantaneous flow rate from centerline velocity in unsteady duct flows, Flow Measurement and Instrumentation, Vol. 19 (2008), pp. 9-15.

[3] Narain, A., Ajotikar, N., Kivisalu, M. T., Rice, A. F., Zhao, M. and Shankar, N., Obtaining time-varying pulsatile gas flow rates with the help of dynamic pressure difference and other measurements for an orifice-plate meter, Transactions of the ASME, Journal of Fluids Engineering, Vol. 135, No. 4 (2013), 041101 . 
[4] Çarpinlioğlu, M. Ö. and Gündoğdu, M. Y., A critical review on pulsatile pipe flow studies directing towards future research topics, Flow Measurement and Instrumentation, Vol. 12 (2001), pp. 163-174.

[5] Mann, J. E., Low cost elbow meter produces accurate readings with low friction loss, Design News, Vol. 25 (1970), p. 56.

[6] Jacobs, G. S. and Sooy, F. A., New method of water measurement by use of elbows in pipe line, Journal of Electricity, Power and Gas, Vol. 27 (1911), pp. 72-78.

[7] Levin, A. M. and Chicago, I., A flow metering apparatus, Transactions of the ASME, Vol. 36 (1914), pp. 239-254.

[8] Addison, H., The use of pipe bends as flow meters, Engineering, Vol. 145 (1938), pp. 227-229.

[9] Ito, H. and Miyakawa, T., Discharge coefficients for 360-deg. bend flowmeters, Bulletin of the JSME, Vol. 121, No. 158 (1978), pp. 1268-1276.

[10] Murakami, M., Oguri, Y., Kato, H. and Sakaida, A., Elbow flowmeters (Effects of wall curvature inaccuracies on discharge coefficients), Bulletin of the JSME, Vol. 21, No. 154 (1978), pp. 697-706.

[11] Mizutani, M. and Oguri, Y., Effects of segmental obstacles at inlet zone on $90^{\circ}$-bend flowmeters, Transactions of the Japan Society of Mechanical Engineers, Ser. B, Vol. 61, No. 588 (1995), pp. 2947-2953 (in Japanese).

[12] Morrison, G. L., Sheth, K. K. and Tatterson, G. B., Elbow flowmeter calibrations for slurries, Chemical Engineering Communications, Vol. 63 (1988), pp. 39-48.

[13] Colwell, J. M. and Shook, C. A., Use of an elbow flowmeter with slurries, Journal of Pipelines, Vol. 7 (1989), pp. 243-250.

[14] Cortelyou, J. T., Centrifugal flow measurement, Journal of SCMA, Instruments \& Control Systems, Vol. 33 (1960), pp. 276-281.

[15] Hothersa11, R. J., Bend meter calibration with unsteady flow, International Journal of Mechanical Engineering Education, Vol. 13, No. 1 (1985), pp. 37-44.
[16] Sumida, M., Pulsatile entrance flow in curved pipes: effect of various parameters, Experiments in Fluids, Vol. 43 (2007), pp. 949-958.

[17] Jarrahi, M., Castelain, C. and Peerhossaini, H., Secondary flow patterns and mixing in laminar pulsating flow through a curved pipe, Experiments in Fluids, Vol.\#50 (2011), pp. 1539-1558.

[18] Sohn, H. C., Lee, H. N. and Park, G. M., A study of the flow characteristics of developing turbulent pulsating flows in a curved duct, Journal of Mechanical Science and Technology, Vol.21 (2007), pp. 2229-2236.

[19] Sumida, M., Senoo, T. and Yamamoto, J., LDV measurements of velocity distribution in unsteady bend flow, International Journal of Mining, Metallurgy \& Mechanical Engineering, Vol. 2, Issue 1 (2014), pp. 21-23.

[20] Kawata, M, Komiya, K. and Yamasaki, H., Handbook of flow measurement, Nikkan Kogyo Shimbun Ltd., (1979), pp. 453-460 (in Japanese).

[21] Tsuji, S. and Kawashima, G., The discharge coefficient of centrifugal-head flow meter, Bulletin of the JSME, Vol. 9, No. 34 (1966), pp. 300-305.

[22] Ito, H., Padjalangi, S. A. and Y. Shoji, Discharge coefficients for 90-, 180- and 360-degs. bend flowmeters (Supplement), Reports of the Institute of High Speed Mechanics, Tohoku University, Vol. 54, No. 452 (1985), pp. 127-152 (in Japanese).

[23] Sumida, M., Sudou, K. and Takami, T., Pulsating flow in curved pipes: 2nd report, experiments, Bulletin of the JSME, Vol. 27, No. 234 (1984), pp. 2714-2721.

[24] Murdock, J. W., Foltz, C. J. and Gregory, C., Performance charcteristics of elbow flowmeters, Transactions of the ASME, Journal of Basic Engineering, Vol. 86, No. 4 (1964), pp. 498-506.

[25] Sumida, M and Sudou, K., Pulsating flow in curved pipes: 3rd report, axial velocity profile, Bulletin of the JSME, Vol. 29, No. 256 (1986), pp. 3334-3340. 